\title{
Non à une régulation durable, alors que les règles actuelles sont ok
}

\section{Jürg Schlup}

Dr, président de la FMH

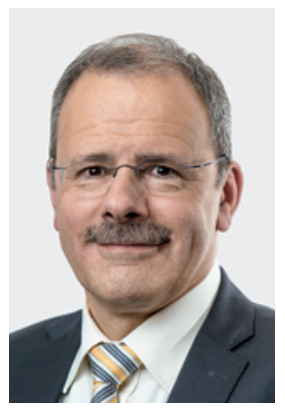

Nul ne conteste que l'évolution des coûts est un des défis majeurs du système de santé de demain. La part des plus de 65 ans, et notamment des plus 80 ans, va s'accroître considérablement ces prochaines années. Dans le même temps, le champ des possibles en médecine va croître et les exigences envers les soins de santé augmenter. L'augmentation de la demande en prestations de santé est donc une certitude.

Au lieu d'élaborer des concepts novateurs pour répondre au mieux à cette évolution, la politique tente depuis 2002 de maîtriser cette demande croissante par une simple limitation des cabinets médicaux ambulatoires - une approche irrationnelle car les médecins en cabinet génèrent seulement $14 \%$ des coûts de santé, et traitent la plus grande partie des maladies. Par ailleurs, la hausse des coûts dans ce secteur a toujours été proportionnelle à l'évolution globale des coûts de la santé, ce qui n'appelle donc aucune intervention particulière.

Il n'est pas étonnant que les tentatives de régulation antérieures soient restées sans effet sur les coûts, même après treize ans de clause du besoin. Le seul résultat obtenu a été un transfert dans la manière de recourir aux prestations et un déplacement des coûts vers le secteur ambulatoire hospitalier, comme le montre leur forte progression depuis 2002.

En dépit de cette absence d'impact sur les coûts, le Conseil fédéral prévoit d'inscrire la régulation des admissions du secteur ambulatoire dans les art. 39 et

Limiter l'offre alors que la demande augmente est une décision irrationnelle qui déplace les coûts au lieu de les réduire.

40 de la LAMal: les cantons pourraient en cas d'«offre excédentaire» - une notion qu'ils devraient définir eux-mêmes - limiter tant les nouvelles admissions de médecins ambulatoires que leur taux d'occupation. Cette disposition multiplierait les conflits d'intérêts liés aux diverses compétences qui relèvent déjà des cantons: autorité de contrôle, formation, propriétaire d'hôpitaux, responsable de la planification hospitalière, promoteur, exploitant, organe de financement, autorité tarifaire et médiateur lors de litiges tarifaires. Si les cantons obtenaient des pouvoirs de régulation pour la médecine ambulatoire, ils auraient par ex. la possibilité de restreindre largement la concurrence face aux services ambulatoires hospitaliers dont ils ont la charge. Mais ils pourraient tout aussi bien ignorer les changements nécessaires dans les hôpitaux comme la création de postes à temps partiel - car les médecins hospitaliers n'auraient de toute façon aucune autre alternative, si ce n'est de renoncer à leur profession. L'exigence de développer des «modèles de soins modernes et novateurs", pourtant soutenue par le Conseil fédéral, serait alors vidée de son sens.

La régulation des admissions proposée ne résout aucun des problèmes actuels et freine des évolutions importantes.

La régulation des admissions telle que proposée ne résout donc aucun des problèmes actuels, freine des évolutions importantes et crée un climat d'incertitude pour la planification des installations de médecins qui voudraient développer leurs propres cabinets. On comprend d'autant moins la volonté du Conseil fédéral d'instaurer une régulation supplémentaire en juillet 2016 que le retour des contingents en février 2017 avec la mise en œuvre de l'initiative dite «sur l'immigration de masse» concernera également le domaine de la santé et les médecins.

Parallèlement, le projet de loi renonce au critère de qualité avéré de la réglementation actuelle, en vigueur depuis 2013, selon lequel sont admis à pratiquer les médecins ayant exercé au moins trois ans dans un établissement de formation postgraduée suisse reconnu. Pourtant, le maintien de cette règle serait acceptable pour la FMH. En effet, il est crucial pour la sécurité des patients que les médecins connaissent bien le système de santé de notre pays et qu'ils disposent d'une formation postgraduée de haute qualité. La FMH rejette à l'unanimité (Comité central et Assemblée des délégués) la proposition de modification de la LAMal et se réserve le droit de lancer un référendum. Si nous souhaitons vraiment préserver la qualité des soins et en garantir le financement, tout doit être fait pour renforcer le domaine ambulatoire et motiver les futurs médecins; et pour cela, il faut créer des mesures incitatives et non ériger des obstacles. 